\title{
APOPTOSIS, OXIDATIVE STRESS AND NEUROLOGICAL DISEASE
}

\author{
Formichi P., Battisti C., Radi E., Di Maio G., Federico A. \\ Department of Neurological and Behavioural Sciences \\ University of Siena
}

Apoptosis is a selective cell deletion process which requires the triggering of a specific cell death programme. Two main pathways determining cell death have been identified: the extrinsic or receptor-mediated pathway, activated in response to extracellular pro-apoptotic signals, and the intrinsic pathway, activated by extracellular receptor-independent stimuli or by intracellular insults, such as DNA damage and oxidative stress. All these stress signals are integrated by mitochondria which participate by releasing the main effectors of this process: a family of aspartic-specific proteases known as caspase. Today there is much evidence to suggest that deregulation of apoptosis is a key feature of many neurodegenerative disease.

Our group sought cell models for the study of apoptotic pathways and for the evaluation of the role of apoptosis in specific neurodegenerative diseases. We focused on oxidative stress-induced apoptosis and activation of the intrinsic mitochondrial pathway. In our in-vitro model, lymphocytes from patients and control subjects were cultured both in basal conditions and with 2-deoxy-D-ribose (dRib), a reducing sugar which induces apoptosis through oxidative stress.

In the last ten years, we evaluated the role of apoptosis in the pathogenesis of several neurodegenerative diseases: Ataxiatelangiectasia, Rett syndrome, Mitochondrial disease, Cerebral autosomal dominant arteriopathy with subcortical infarcts and leukoencephalopathy (CADASIL). Here we report some of our ongoing and recently published articles.

Apoptosis is a selective cell deletion process which requires the triggering of a specific cell death programme. Apoptosis is necessary for the elimination of redundant cells and for tissue remodelling during embryo development in invertebrates and vertebrates. The exact apoptotic pattern is crucial for the development of certain organs and for differentiation of the nervous and immune systems (1). The apoptotic process is also essential for the maintenance of cellular homeostasis in adults, especially adult mammals, which during their long lives are exposed to many pathological signals which may induce cell death (2). Deregulation of apoptotic pathways may be a primary event in the onset of various diseases, since very high and very low apoptotic levels can lead to degenerative and proliferative pathologies, respectively (3).

It has been shown that pathologies such as autoimmunity and cancer are related to a deficit in apoptotic mechanisms, while an increase in apoptotic levels may determine acute and chronic degenerative diseases, such as those of the nervous system, ischemic diseases, immunodeficiency and autoimmunity (3).

Cells undergoing apoptosis may be identified by numerous morphological modifications that can be detected by light and electron microscopy; these modifications are surprisingly similar in different cell types and species and are irrefutable evidence of an ongoing process (1). Apoptotic cells are characterized by loss of contact with neighbouring cells, chromatin condensation, cell shrinkage and blebbing of the plasma membrane with formation of "apoptotic bodies" (4). Apoptotic bodies are membranebounded cell fragments, containing portions of nucleus and intact organelles, which are recognized and engulfed by tissue macrophages without any inflammatory reaction (Fig. 1).

The apoptotic process and clearance of apoptotic cells are so rapid in vivo (a few hours at most) that identification

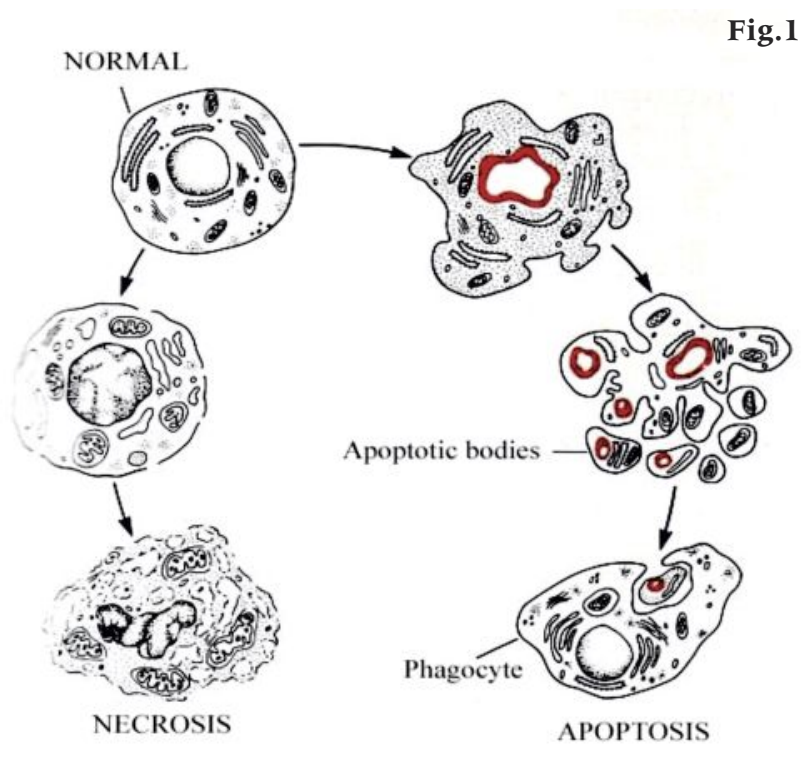

of only a few apoptotic bodies in a tissue section may indicate a significant degree of cell loss and can be considered an important biological phenomenon (5).

In the last few decades, many studies have attempted to clarify the signalling mechanisms leading to apoptosis and have identified two main pathways determining cell death: the extrinsic and intrinsic pathways.

The extrinsic or receptor-mediated pathway is activated in response to extracellular pro-apoptotic signals and integrated into the apoptotic process via specific cell receptor adaptors (6). This pathway is crucial for tissue homeostasis and immune system integrity (Fig. 2).

The intrinsic pathway is activated by extracellular receptor-independent stimuli (gamma and UV rays, hypoxia, nutrient and growth factor deficiency) or by intracellular insult, such as DNA damage and oxidative stress (2). All these stress signals are integrated by mitochondria, which participate by releasing pro-apoptotic factors. Permeabi-

\section{Correspondence to:}

Prof. Antonio Federico. Department of Neurological Sciences and Behaviour, Siena University, Italy.

Policlinico "Le Scotte" - Viale Bracci - 53100 - Siena - Italy.

Tel +390577585763- +390577263355 Fax +39057740327

e-mail: federico@unisi.it 
Fig.1 (left): Morphological alterations in apoptotic and necrotic cell death.
Fig.2 (right): Extrinsic and intrinsic apoptotic pathway and their interactions.

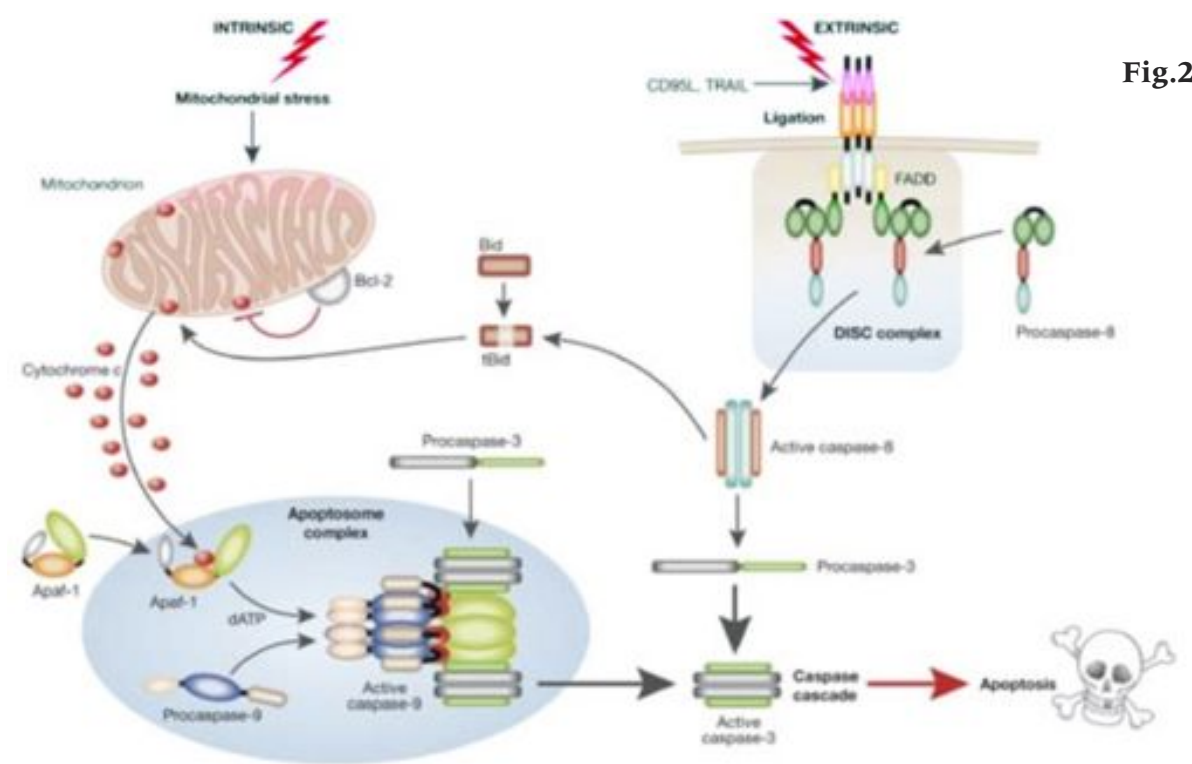

lization of the outer mitochondrial membrane is a key step in apoptosis, although its mechanism has not been completely clarified. One model of this process involves a change in membrane permeability (mitochondrial permeability transition MTP) by opening of permeability transition pores (PTP), resulting in a decrease in mitochondrial membrane potential $(\Delta \psi \mathrm{m})$. It has been shown that this mechanism is influenced by high intracellular calcium concentrations (7). The extrinsic and intrinsic pathways converge in apoptotic process, highly conserved through evolution. The main effectors of this process is a family of aspartic-specific proteases known as caspase $(8,9)$ and the major biochemical markers of which are nuclear DNA fragmentation and exposure of phosphatidylserine (PS) on the outer cell membrane (9) (Fig. 2).

The existence of a third apoptotic pathway activated by the accumulation of misfolded protein in the endoplasmic reticulum (ER) was recently demonstrated. ER stress conditions trigger the unfolded protein response (UPR) which activates several target genes and determines translation of regulatory proteins restoring normal "protein homeostasis". However, when the stress is irreversible, UPR may activate caspase and promote apoptosis. Apoptotic cell death is a normal regressive event in development of the central nervous system (CNS) and indeed about 50\% of post-mitotic neurons die by apoptosis during embryogenesis (11).

It is also now clear that apoptosis in neurons mainly follows the intrinsic pathway as caspase 3- and 9-deficient mice show severe brain malformations due to lack of neuronal apoptosis (12). Altered regulation of apoptosis is implied in the progression of many neurological diseases: in acute neurological diseases, necrosis and caspase-mediated apoptosis occur simultaneously; in chronic neurodegenerative diseases, caspase-mediated apoptotic pathways mediate cell dysfunction and death (13).

Today there is much evidence to suggest that deregulation of apoptosis is a key feature of many neurodegenerative disease like Alzheimer's disease (AD), spinal muscular atrophy (SMA), Huntington disease (HD), amyotrophic lateral sclerosis (ALS) and Parkinson disease (PD). It is thus clear that apoptosis has a determinant role in the evolution of neurodegenerative diseases. Data in the literature shows that neuronal apoptosis is an extremely complex process, making study of apoptosis in neurodegeneration very important for understanding the pathogenesis of diseases and identifying new therapeutic targets.

In recent years, our group has sought cell models for the study of apoptotic pathways and for evaluation of the role of apoptosis in specific neurodegenerative diseases. Since most of the work on the role of apoptosis in neurodegeneration has been based on animal models, our first aim was to use cells from patients in our experiments. We focused on oxidative stress-induced apoptosis and activation of the intrinsic (mitochondrial) pathway. We used quiescent cells such as lymphocytes as cell model, treating them with 2-deoxy-D-ribose (dRib), a reducing sugar which induces apoptosis through oxidative stress due mainly to glutathione depletion (14). We also tested many other cell types, such as lymphoblasts, fibroblasts and microglial cells.

In our in-vitro model, cells cultured under basal conditions and treated with dRib were collected at different times and analyzed qualitatively and quantitatively by various methods. We treated cells with specific dyes and studied them by light and fluorescence microscopy for:

1) DNA fragmentation by Hoechst H33258;

2) caspase 3 and 7 activation by Fluorochrome Inhibitor of Caspases (FLICA)(15);

3) dissipation of mitochondrial membrane potential by a mitochondrion specific probe of the cabocyanin family: 5, 5'6, 6' -tetrachlorol, 1',3,3' - tetraethylbenzimidazolcarbocyanine iodide (JC-1) (16);

4) externalization of phosphatidylserine (PS). During apoptosis, PS translocates from the inner to the outer layer of the plasma membrane and apoptotic cells can be detected by Annexin V binding to translocated membrane PS (17).

We also used:

1) agarose gel electrophoresis, which identifies nuclear DNA fragmentation through characteristic apoptotic ladder pattern (Fig. 3) (18); 
Fig.3: Agarose gel electrophoresis of DNA from a control subject (a) and an AT patient (b).

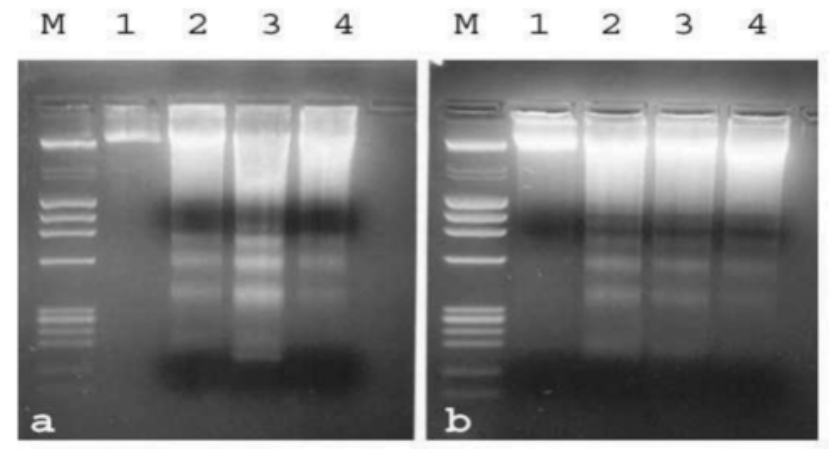

2) flow cytometry for quantitative measurement of apoptosis by reduced fluorescence of the propidium iodide (a DNA binding dye) (19);

3) in situ hybridization by terminal deoxynucleotidyl transferase mediated dUTP nick and labelling (TUNEL), which detects apoptotic cells in autopsy and biopsy samples of brain, muscle and skin.

\section{APOPTOSIS AND NEUROLOGICAL DISEASE}

In the last ten years, our group validated and used the above cell models and analytical methods to evaluate the role of apoptosis in the pathogenesis of certain neurodegenerative diseases. Here we report some of our ongoing and recently published articles.

\section{Ataxia telangiectasia}

Ataxia-telangiectasia (AT) is a rare autosomal recessive disease characterized by cerebellar degeneration, growth retardation, diffuse ocular and auricular telangiectasia, premature ageing, high incidence of malignant tumours, especially lymphatic cancer, and immunodeficiency. Hypersensitivity to radiation and chromosome instability are the biological markers of this disease. ATM, the gene responsible for AT, was identified on chromosome 11q2223 and encodes a protein involved in several cell responses to growth factors and DNA damage. ATM protein is involved in the regulation of apoptosis.

On the basis of these findings, our group evaluated the role of the ATM gene in the regulation of the cell cycle and of apoptosis, using peripheral blood lymphocytes (PBLs) and lymphoblasts from AT patients and controls exposed to apoptotic stimuli. Cells were exposed to $10 \mathrm{mmol} / \mathrm{L}$ dRib and harvested after 1, 24, 48 and 72 hours of culture. Pellets were analysed by flow cytometry and agarose gel electrophoresis. Our results showed an impaired response to dRib-induced apoptosis in AT cells (Fig. 3), as well as a defect in cell cycle arrest in G1/S phase and normal expression of p53 protein. These results suggested a defect in the regulation rather than in the expression of $\mathrm{p} 53$ protein and indicate that the kinase activity of the ATM product plays a very important role in this regulation as well as in cell response to oxidative stress (20).

Finally, the evident resistance to apoptotic cell death observed in AT cells could explain their susceptibility to malignant transformation.

\section{Rett syndrome}

Rett syndrome (RS) is a neurological disorder that primarily affects females. In 1999 Amir et al., identified mutations in a gene encoding $\mathrm{X}$-linked methyl CpG binding protein 2 (MeCP2) as the cause of most cases. However, the pathogenesis of this syndrome is still unknown. It was recently shown that RS is a neurodevelopmental disorder. Brain hypoplasia, dendritic branching decrease, dendritic spine derangements and other synaptic abnormalities indicate disordered organization of neuronal networks during post natal life and strongly support the hypothesis of specific brain growth factor deficiency or apoptotic defect. In order to clarify the role of apoptosis in this disorder, we studied lymphoblastoid cell lines. Lymphoblasts from RS patients under basal condition showed a lower percentage of apoptosis than those of controls, whereas in the presence of dRib, the percentage of apoptotic cells in RS patients increased with time and reached the same percentage as controls after $72 \mathrm{~h}$. These results suggested that RS may have low susceptibility or increased resistance to oxidative stress-induced apoptosis, which may only be corrected by a strong apoptotic stimulus (21).

\section{Mitochondrial disease}

Mitochondria play a key role in programmed cell death: i) they release cytochrome $c$ into the cytosol in the first phase of apoptosis, activating caspase 9; ii) the process seems to be inhibited by bcl-2, a protein belonging to a large family of proteins involved in apoptosis located in the outer mitochondrial membrane; iii) the opening of mitochondrial permeability transition pores is an early event in apoptosis; iv) electron transport alterations, oxidative phosphorylation dysfunction and storage of free radicals (all suggestive of mitochondrial dysfunction) have been demonstrated in apoptotic cells (22).

On the basis of these findings we investigated the role of oxidative stress-induced apoptosis in patients with mitochondrial diseases, particularly in patients with Leber's hereditary optic neuropathy (LHON). Previous research showed that death of retinal ganglion cells characteristic of LHON occurs through apoptosis, so that changes in the mitochondrial respiratory chain and/or in oxidative stress could play a role in induction of apoptosis in LHON.

We analysed PBLs from LHON patients and control subjects incubated with dRib, finding a higher rate of apoptosis in cells of patients than controls. We also found evidence of mitochondrial involvement in activation of the apoptotic cascade (Fig. 4).

Our results showed that LHON cells are particularly susceptible to oxidative stress-induced apoptosis and confirmed a direct link between complex I (commonly altered in patients with LHON) and changes in mitochondrial membrane permeability (23).

We also evaluated the role of apoptosis in the pathogenesis of other mitochondrial diseases using the TUNEL technique. We looked for apoptosis in muscle biopsies from 17 patients with mitochondrial encephaloneuromyopathies, such as MERRF, MELAS, cPEO and MENM. Our results showed few apoptotic (TUNEL-positive) nuclei in the biopsies, except in MERRF patients, and no relationship between respiratory chain defect and 
percentage of apoptotic myonuclei. However, the findings were very heterogeneous, even between patients with the same mtDNA mutations, suggesting that in mitochondrial pathologies, tissue evaluation of apoptosis is less useful than in vitro techniques (24) (Fig. 5). Finally we looked for apoptotic nuclei in autopsy brain tissue from a patient with familial Leigh syndrome, by the TUNEL method. We observed numerous TUNEL-reactive neuronal cells showing morphological signs typical of apoptosis in cortical areas suffering neuronal cell loss. Our findings suggested that apoptosis may be involved in the mechanism of neuronal death in Leigh syndrome (25) (Fig. 6).

\section{CADASIL}

Cerebral autosomal dominant arteriopathy with subcortical infarcts and leukoencephalopathy (CADASIL) is an autosomal dominant cerebrovascular disease leading to cognitive decline and dementia, characterized by migraine with aura and recurrent ischemic strokes (26). The pathologic hallmark of CADASIL is systemic non-atherosclerotic vasculopathy, which predominantly affects the small cerebral arteries through progressive degeneration of vascular smooth muscle cells (VSMC). Accumulation of granular osmiophilic material (GOM) has been observed in the walls of small vessels in the brain, skin, muscle and peripheral nerves.

CADASIL is caused by single missense mutations, or more rarely by small deletions, in the Notch3 gene which encodes Notch3 protein, a member of a conserved transmembrane receptor family (Notch signalling) (27). Notch 3 is mainly expressed in VSMC, though it is claimed to be expressed ubiquitously in adult human tissues (28).

The pathogenetic mechanism leading to CADASIL is not yet completely understood. There is evidence to suggest that apoptosis may be involved in VSMC degeneration and loss (29). Recent studies have shown that Notch3 signalling in VSMC plays an important role in down-regulating Fas-Ligand (FasL)-induced apoptosis (extrinsic apoptotic pathway) (30). Involvement of Notch3 in the regulation of apoptosis has also been observed in other cell types, such as lung carcinoma cells, where inhibition of the Notch3 signalling pathway mitigates the neoplastic phenotype (31). Finally, Viswanathan et al. (2006) found widespread neuronal apoptosis in the cerebral cortex of CADASIL brains, demonstrating that the degree of neuronal apoptosis is related to the underlying subcortical white matter damage (32). On the basis of these findings we evaluated the role of apoptosis in the

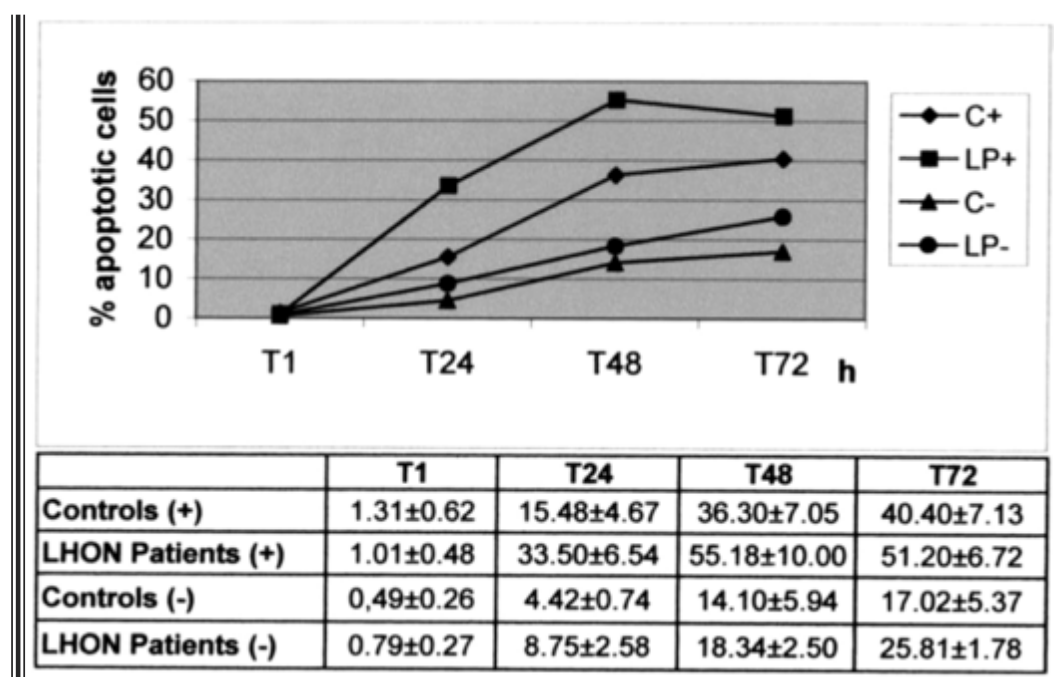

Fig.4 (above):

Cytofluorimetric analysis of apoptotic PBLs of eight patients with LHON (LP) and six controls (C) cultured with (+) and without (-) dRib. Cells were analysed after 1 (T1), 24 (T24), 48 (T48) and 72 (T72) hours of culture. Values are expressed as mean percentage $( \pm \mathrm{SD})$ of apoptotic PBLs. a)
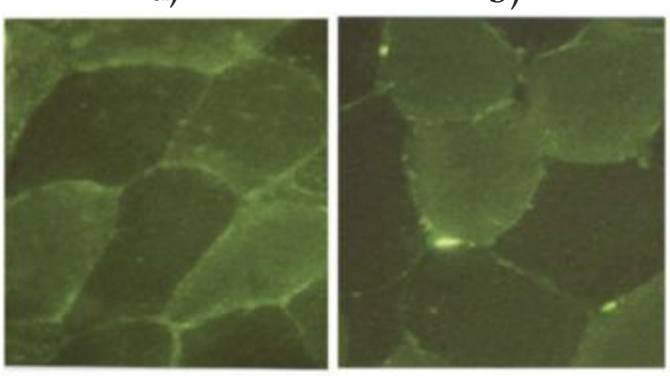

Fig. 5 (above):
TUNEL reaction in normal (a), mitochondrial myopathic (MELAS) (b) and DNAse I treated (c) muscle. Sections were analyzed under fluorescence microscope. TUNEL-positive nuclei are shown in greenish-yellow. (40X)

Fig.6 (below):

Section of brain tissue from patient with Leigh syndrome analysed by TUNEL assay. Brown nuclei are TUNEL positive.

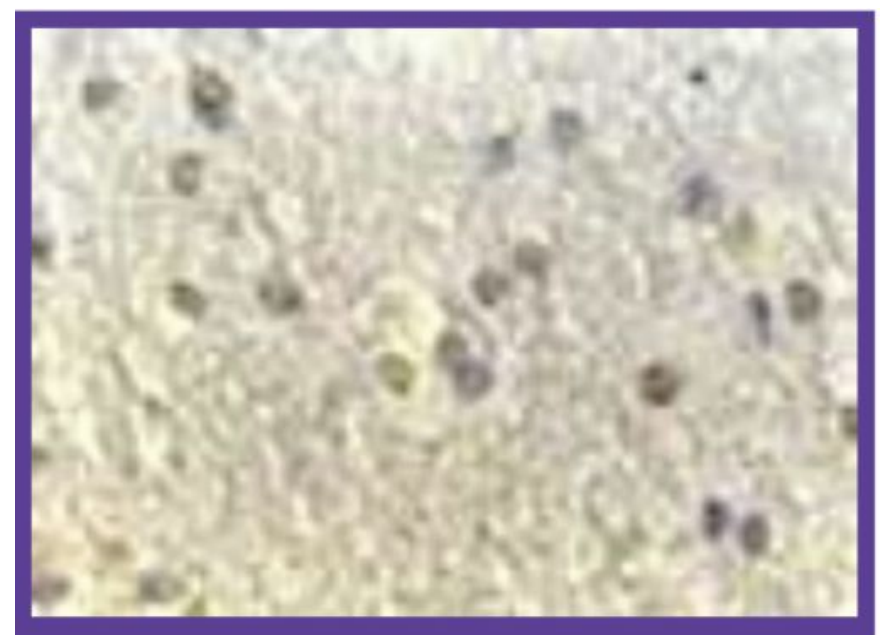


pathogenesis of CADASIL by assessing the apoptotic response of peripheral blood lymphocytes (PBLs) and fibroblasts from CADASIL patients exposed to 2-deoxy-D-ribose (dRib), a reducing sugar which induces apoptosis through oxidative stress, and also assessing the extent of neuronal apoptosis in a young autopsy case of CADASIL.

\section{In-vitro study}

PBLs from CADASIL patients showed a higher degree of apoptosis than controls, both under standard conditions and after incubation with dRib. After 72 hours of culture under standard conditions, PBLs from patients showed an elevated percentage of apoptotic cells $(6.18 \%)$, three times higher than that of controls $(1.98 \%)$. After $72 \mathrm{~h}$ of incubation with dRib, the percentage of apoptotic cells in CADASIL patients $(31.21 \%)$ was twice that of controls (16.51\%) (Fig. 7). The results of cytofluorimetric analysis were confirmed by agarose gel electrophoresis and fluorescence microscopy.

For fibroblasts cultured under standard conditions, fluorescence microscopy analysis did not show any significant difference in the percentage of apoptotic cells between patients and controls. After $48 \mathrm{~h}$ of dRib treatment a higher percentage of apoptotic fibroblasts was evident in CADASIL than control cultures.

The first result of our study was that cells of CADASIL patients showed major apoptosis after dRib treatment, which demonstrates that CADASIL cells are particularly sensitive to oxidative stress-induced apoptosis, possibly related to the presence of Notch 3 mutations. Although most data in the literature demonstrates that Notch 3 is involved in protective mechanisms against the extrinsic apoptotic pathway, our results suggest that Notch 3 may also be implicated in the regulation of the intrinsic apop- totic pathway. Our most striking result was that even under standard culture conditions, PBLs from CADASIL patients showed an elevated degree of apoptosis, suggesting that cells carrying a mutation in the Notch 3 gene may be subject to a higher level of intracellular stimuli or insults than normal cells. In particular, CADASIL cells may have an altered redox state and accumulate reactive oxygen species (ROS), leading to a high basal level of apoptosis and to increased susceptibility to external stimuli such as oxidative stress. This hypothesis is in line with evidence of a relationship between Notch3 gene mutations, impairment of mitochondrial function and stress of endoplasmic reticulum (ER) $(33,34)$.

\section{TUNEL assay}

We analyzed seven-micron-thick paraffin embedded sections of temporal lobe, brainstem, bulb, hippocampus and basal ganglia from the brain of a female CADASIL patient, deceased at 41 years of age. Many apoptotic nuclei were detected among glial cells in temporal lobe, bulb, basal ganglion and hippocampus specimens $(28 \%, 33 \%, 30 \%$, $27 \%$ respectively), while neurons seemed unaffected. On the other hand, widespread neuronal apoptosis $(60 \%)$ was observed in brainstem, especially the pons-mesencephalus region, where a high percentage of apoptotic glial cells was also evident (34\%) (Fig. 8 a, b, c, d, e).

The brain regions analyzed in this study showed a significant number of cells involved in apoptotic processes, especially glial cells. Apoptotic neurons were only found in the brainstem, seeming to be spared in the other areas examined. The apoptotic study confirmed the results of neuropathological examination and brain NMR, where cortical neurons seemed relatively uninvolved in ischemic lesions. Our finding of diffuse apoptosis of glial cells but not of cortical neurons seems at variance with data in the

\section{CADASIL and dRib-induced apoptosis}

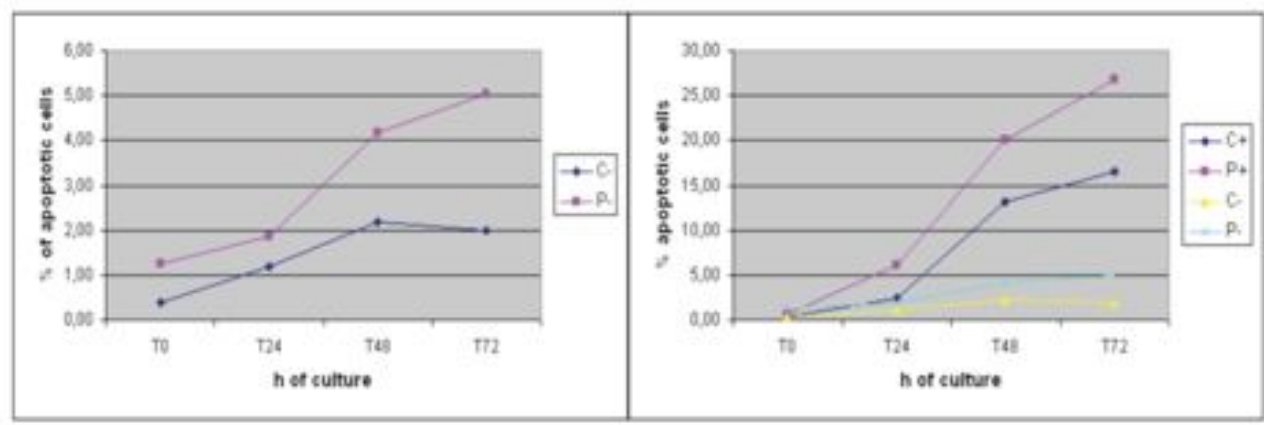

Fig.7:

Cytofluorimetric analysis of PBLs from 16 CADASIL patients $(\mathrm{P})$ and controls (C after 1 (T1), 24 (T24), 48 (T48) and 72 (T72) h of incubation without (-) and with (+) dRib

\begin{tabular}{|c|c|c|c|c|}
\hline \multicolumn{5}{|c|}{ Table 1} \\
\hline & To & T24 & T48 & T72 \\
\hline Con- (4) & $0,38 \pm 0,18$ & $1,20 \pm 0,47$ & $2,20 \pm 0,43$ & $1,98 \pm 0,91$ \\
\hline Con+ (4) & $0,39 \pm 0,22$ & $2,39 \pm 1,86$ & $13,14 \pm 1,86$ & $16,51 \pm 1,97$ \\
\hline $\begin{array}{l}\text { CADASIL- } \\
\text { (12) }\end{array}$ & $1,25 \pm 0,98$ & $1,87 \pm 0,94$ & $4,19 \pm 1,67$ & $5,04 \pm 2,31$ \\
\hline $\begin{array}{l}\text { CADASIL. } \\
\text { (12) }\end{array}$ & $0,65 \pm 0,44$ & $6,02 \pm 5,89$ & $19,98 \pm 8,67$ & $26,79 \pm 8,48$ \\
\hline
\end{tabular}


literature (32). Since our patient was young (41 years) and had slight mental deterioration, the divergence may be related to the different ages at which apoptosis was evaluated, as well as to the degree of cognitive impairment. Our findings suggest that in the early phases of CADASIL, apoptosis may be restricted to subcortical ischemic regions and to brainstem neurons, in line with several clinical and neuroradiological studies of affected patients. Apoptosis may only involve cortical neurons, superior functions and loading in later stages of the disease. Results of clinical and histopathological examination may be at variance in CADASIL patients of different ages or in different stages of the disease.

In conclusion, these results indicate impaired regulation of apoptosis in CADASIL cells and suggest that the Notch3 gene may play a key role in regulation of the intrinsic apoptotic pathway. Moreover, the presence of numerous TUNEL-positive cells in brain tissue of CADASIL patients, suggests that apoptosis may have an important role in the pathogenesis of the disease.

\section{BibliografiA}

1. Kerr Jf, Wyllie Ah, Currie Ar. Br J Cancer 26: 239-257, 1972.

2. Danial Nn, Korsmeyer Sj. Cell 116:205-219,2004.

3. Fadeel B, Zhivotovsky B, Orrenius S., Faseb J 13: 16471657,1999 .

4. Guimaraes Ca, Linden R. Eur J Biochem 271: 1638-1650, 2004 5. Fadeel B, Orrenius S, Zhivotovski B. Biochem Biophys Res Comm 266: 699-717, 1999.

6. Kutuk O, Basaga H. Apoptosis 11:1661-1675, 2006.

7. Gogvadze V, Orrenius S, Zhivotovsky B. Biochem Biophys Acta 1757:639-647, 2006.

8. Hengartner Mo. Nature 407:770-776, 2000

9. Zhivotovsky B, Burgess Dh, Vanags Dm, Orrenius S. Biochem Biophys Res Comm 230:481-488, 1997.

10. Bredesen De. In: Tomei Ld, Cope Fo, Eds Apoptosis Ii: The Molecular Basis Of Apoptosis In Disease. Pailview, Ny: Cold Spring Harbor Laboratry Press, 397-421,1993.

11. Lo Ac, Houenou Lj, Oppenheim Rw. Arch Histol Cytol 58:139$149,1995$.

12. Ranger Am, Malynn Ba, Korsmeyer Sj. Nat Genet28:113-118, 2001.

13. Friedlander Rm. N Engl J Med 348:1365-1375, 2003.

14. Kletsas D, Barbieri D, Stathakos D, Botti B, Bergamini S, Tomasi A, Monti D, Malorni W, Franceschi C. Biochem Biophys Res
Commun. 243(2):416-25, 1998.

15. Ekert Pg, Silke J, Vaux Dl. Cell Death Differ.; 6:1081$1086,1999$.

16. Lieven Cj, Vrabec Jp, Levin La.. Antioxid Redox Signal.; 5:641646, 2003.

17. Vermes I, Haanen C, Steffens-Nakken H, Reutelingsperger C. J Immunol Methods. 184:39-51, 1995.

18. Formichi P, Battisti C, Tripodi Sa, Tosi P, Federico A. Life Sci., 66(20):1893-903, 2000.

19. Nicoletti I, Migliorati G, Pagliacci Mc, Grignani F, Riccardi C. J Immunol Methods, 139:271-279, 1991

20. Battisti C, Formichi P, Tripodi Sa, Mangiavacchi P, Tosi P, Federico A. J Neurol Sci 144: 128-134, 1996.

21. Battisti C, Formichi P, Tripodi Sa, Meloni I, Mangiavacchi $P$, Zappella M, Federico A. Brain \& Development 26:384-388, 2004 22. Li N, Ragheb K, Lawler G, Sturgis J, Rajwa B, Melendez Ja, Robinson Jp.J Biol Chem, 278(10):8516-25, 2003.

23. Battisti C, Formichi P, Cardaioli E, Bianchi S, Mangiavacchi $P$, Tripodi Sa, Tosi P, Federico A. J Neurol Neurosurg Psychiatry, 75: 1731-1736, 2004.

24. Formichi P, Battisti C, Bianchi S, Cardaioli E, Federico A. J Submicrosc Cytol Pathol, 35(1):29-34, 2003.

25. Formichi P, Malandrini A, Battisti C, Santorelli Fm, Gambelli S, Tripodi Sa, Berti G, Salvadori C, Tessa A, Federico A. J Neurol Neurosurg Psychiatry, 75: 930-932, 2004.

26. Ruchoux Mm, Guerouaou D, Vandenhaute B, Pruvo Jp, Vermesh P, Leys D. Acta Neuropathol, 89:500-512, 1995.

27. Tournier-Lasserve E, Joutel A, Melki J, Weissenbach J, Lathrop Gm, Chabriat H, Mas J, Cabanis Ea, Baudrimont M, Maciazek J, Bach Ma, Bousser Mg. Nature Genet, 3:256-259, 1993.

28. Joutel A, Andreux F, Gaulis S, Domenga V, Cecillon M, Battail N, Piga N, Chapon F, Godfrain C, Tornier-Lasserve E. J Clin Invest, 105 :597-605, 2000

29. Gibbons Gh, Pollman Mj. Circ Res. 86, 1009-1012, 2000

30. Wang W, Prince Cz, Mou Y, Pollman Mj. J Biol Chem, 277, 21723-21729, 2002.

31. Haruki N, Kawaguchi Ks, Eichenberg S, Massion Pp, Gonzalez A, Gazdar Af, Minna Jd, Carbone Dp, Dang Tp. Cancer Res. 65, 3555-561, 2005.

32. Viswanathan A, Grey $F$, Bousser Mg, Baudriumont M, Chabriat H. Stroke, 37(11):2690-2695, 2006.

33. De La Peña, P., Bornstein, B., Del Hoyo, P., Fernández-Moreno, M.A., Martín, M.A., Campos, Y., Gómez-Escalonilla, C., Molina, J.A., Cabello, A., Arenas, J., Garesse, R., 2001. Mitochondrial Dysfunction Associated With A Mutation In The Notch3 Gene In A Cadasil Family. Neurology 57, 1235-1238.

34. Ihalainen, S., Soliymani, R., Iivanainen, E., Mykkänen, K., Sainio, A., Pöyhönen, M., Elenius, K., Järveläinen, H., Viitanen, M., Kalimo, H., Baumann, M., 2007. Proteome Analysis Of Cultivated Vascular Smooth Muscle Cells From A Cadasil Patient. Mol. Med. 13, 305-314.

Fig.8:

TUNEL staining of paraffin embedded sections from CADASIL patient (brown nuclei are TUNEL positive). In temporal lobe (a), bulb (b), basal ganglia (c) and hippocampus (d) sections, widespread apoptosis of glial cells is evident (20x). In the brain stem region (e), numerous apoptotic neurons are evident (arrows) (40x).

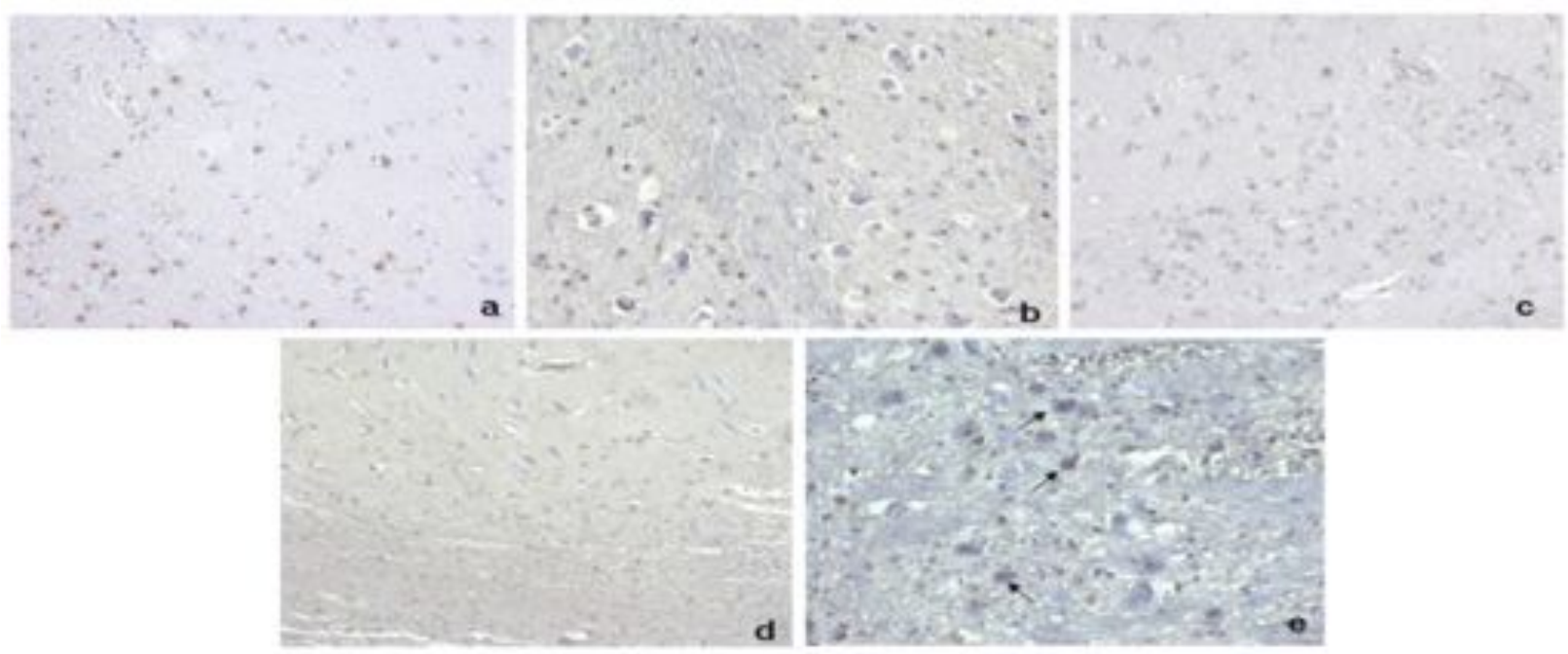

